\title{
Le tutorat dans l'enseignement à distance, un geste pédagogique
}

\author{
Françoise Greffier
}

Laboratoire d'Informatique de Franche-Comté (LIFC)

UFR Sciences et Techniques

16 , route de Gray

F-25030 Besançon cedex

greffier@lifc.univ-fcomte.fr

RÉSUMÉ. Cet article résulte d'une expérience de tutorat exercé dans le cadre d'une licence d'informatique réalisée à distance via Internet. Dans ce contexte, nous abordons l'industrialisation du tutorat sous l'angle de la création d'outils informatisés pour les apprenants et leur tuteur. Nous montrons en quoi il est essentiel que les outils informatisés intègrent des fonctionnalités soutenant les apprenants dans leur processus d'acquisition des connaissances. Nous présentons un exemple d'outil conçu dans cet esprit : une application informatisée incluant une base de données d'exercices. Nous soulignons qu'un bon couplage du geste pédagogique accompli par le tuteur avec l'utilisation des TIC est un facteur de qualité dans l'enseignement à distance. Ce facteur de qualité est exprimé aussi en termes de limites à une industrialisation du tutorat.

ABSTRACT. This article reports a learner support experience led as part of an on line distance course for a third-year university degree in Computer Science. We tackle tutoring "industrialisation" by means of the creation of computer tools for learners and their tutors. We show why it is fundamental for such computer tools to include functions that help learners in their learning process. We describe an application including a database of exercises as an example of a tool designed within this framework. We emphasise the idea that a good coupling between the use of ICT and the teaching skills provided by tutors is a quality factor for e-learning. This coupling could also show the limits of tutoring industrialisation.

MOTS-CLÉS : formation en ligne, apprentissage collaboratif, tutorat, industrialisation, qualités pédagogiques.

KEYWORDS: on line learning, collaborative learning, tutoring, industrialisation, teaching skills.

D\&S - 3/2005. Tutorat et logiques industrielles, pages 231 à 250 


\section{Introduction}

Cette contribution est le fruit d'un intérêt pour la pédagogie et de réflexions nées d'une expérience de tutorat, menée depuis cinq ans, dans le cadre d'un enseignement à distance. Cet enseignement concerne un module de programmation pour une licence informatique dispensée complètement à distance via Internet.

D'une façon générale, nous cherchons à améliorer notre pratique de tuteur, à partager des idées et à concevoir et implanter des logiciels pour l'apprentissage utiles et testables dans le contexte d'enseignement évoqué ici.

Pour cet article, nous abordons l'industrialisation du tutorat sous l'angle de la conception de «machines pour apprendre», machines reproductibles dans tout contexte d'enseignement à distance. Compte tenu des moyens actuels liés aux nouvelles technologies, nous considérons des supports d'enseignements informatisés et véhiculés sur Internet.

Afin de réfléchir aux possibilités et limites de l'industrialisation du tutorat, la première question est celle de l'existence ou non de modèles généralisables, c'est-àdire fondés sur des processus reproductibles d'acquisition des connaissances.

Dans l'optique d'obtenir des «machines pour apprendre » le plus largement informatisées possible, nous posons également la question de savoir si l'ensemble de l'activité du tuteur est industrialisable, ou bien si elle ne l'est que partiellement. Pour apporter des éléments de réponse, il est utile de revenir sur le métier de tuteur. Quelles sont les conditions de réussite de l'apprentissage à distance liées à la spécificité de ce type d'enseignement? Pour satisfaire au mieux ces conditions, quels sont les fonctions et rôles majeurs du tuteur? Quelles compétences méthodologiques sont requises pour un tutorat de qualité ? Les fonctions tutorales étant liées à la constitution du groupe et à ses objectifs, ces derniers sont précisés également.

Tout en discutant des limites de l'industrialisation du tutorat, nous développons l'idée selon laquelle la fabrication d'outils informatisés pour l'apprentissage est de plus un atout pour la réussite de l'enseignement à distance. Un exemple d'outil est présenté pour étayer ces propos : il s'agit d'un exerciseur conçu et implanté pour un module de programmation d'une licence à distance. Cette présentation met en relief des principes et fondements pour concevoir des outils informatisés de qualité qui soient adaptés aux particularités de l'enseignement à distance.

Nous montrons que, pour être performante et réaliste, une industrialisation du tutorat est à envisager sur la base d'un couplage efficace des possibilités offertes par les nouvelles technologies et de l'apport humain exprimé par le tuteur, les apprenants et le groupe. 


\section{Tutorat pour une licence informatique à distance via Internet}

Notre expérience de tutorat est réalisée dans le cadre d'un enseignement à distance complètement assisté par Internet. En effet, toutes les activités d'apprentissage s'y effectuent à distance via Internet et, pour l'ensemble de ces activités, les apprenants bénéficient d'un accompagnement par un tuteur.

Il est important de comprendre le contexte dans lequel s'inscrit cette réflexion car il est en lien direct avec les points de vue adoptés. A cet effet, nous présentons brièvement cette formation à distance puis les caractéristiques du groupe d'apprenants. Nous reviendrons ensuite sur les facteurs de qualité d'une formation et sur le rôle du tuteur.

\section{La licence informatique à distance}

Cette formation délivre une licence en informatique préparée en deux ans. Elle existe depuis cinq ans et regroupe une soixantaine d'apprenants par promotion. Ces derniers sont recrutés sur dossier. Chaque apprenant a la possibilité de suivre quatre modules par an, soit deux par semestre, un semestre couvrant douze semaines d'apprentissage.

Le module dans lequel nous intervenons forme les apprenants à la programmation orientée objet. Nous sommes auteur des contenus du module: connaissances et exercices. Ces supports sont écrits en HTML (liens hypertexte). Chaque séquence d'apprentissage (sur douze semaines) se déroule avec la participation d'un tuteur dont nous assurons la fonction depuis cinq ans. Il n'y a pas d'autre tuteur pour accompagner ces apprenants.

\section{Objectif de l'enseignement}

Le module enseigné s'intitule «Méthodes et Outils pour la Programmation orientée Objet ».

Le libellé du module montre bien que l'essentiel n'est pas d'apprendre un outil de programmation orientée objets (le langage de programmation $\mathrm{C}++$ ), même si nous avons choisi ce langage pour illustrer le cours et programmer les exercices. Il s'agit pour l'apprenant d'intégrer véritablement une méthode de programmation. Cette méthode peut bousculer la façon de penser appliquée jusque-là dans les activités de programmation, car elle propose une autre façon de modéliser le monde (par objets). Il s'agit donc, pour certains apprenants, d'intégrer des nouvelles représentations des connaissances.

\section{Le groupe}

Comment se profile ce groupe d'apprenants? Par rapport à leur projet professionnel, il y a des apprenants en reconversion ou en consolidation de savoirs, 
savoir-faire... d'autres en reprise d'études (suite à un arrêt des études à bac +2 ). Leurs âges vont de vingt à cinquante ans. Leur degré de maturité et leur expérience dans l'apprentissage ou dans la programmation sont donc variables.

La programmation exige des prérequis importants. Or, environ un étudiant sur cinq n'a pas complètement intégré ces prérequis. Le niveau est très hétérogène car, par ailleurs, certains apprenants (4 à 5 par promotion) connaissent déjà bien la matière enseignée.

Leur inscription à une formation délivrant un diplôme nous laisse penser qu'ils partagent un but commun : obtenir un maximum de points au module. Il se peut que certains viennent par curiosité et ne passent pas les examens, mais nous constatons que cette démarche reste marginale.

Suite à ces observations, nous résumons les caractéristiques des étudiants et de cet enseignement en quelques points :

- les apprenants poursuivent un même but et ont un intérêt commun, celui d'obtenir la licence et donc d'avoir une bonne note au module ;

- le niveau en programmation est hétérogène ;

- les enjeux peuvent être plus ou moins importants. Certains comptent sur la réussite dans cette formation pour trouver ou garder un emploi ;

- nous considérons le groupe comme un groupe d'adultes composé des apprenants et du tuteur ;

- les échanges s'effectuent par écrit (courriels, participation au forum de discussion, publications de pages html).

\section{Un groupe d'adultes qui augmente ses chances de réussite}

La question est de savoir quels sont les facteurs, au niveau individuel comme au niveau collectif, qui favorisent la réussite de l'apprentissage. Ces facteurs sont ensuite repris pour définir le rôle du tuteur et dégager les capacités requises pour réaliser un tutorat de qualité.

\section{Autonomie et interdépendance}

Reprenons une des caractéristiques du groupe : il est composé d'adultes. Cela signifie que les apprenants ont un degré d'autonomie suffisant pour gérer leur apprentissage en interdépendance avec les autres membres du groupe. Pour nous, l'interdépendance signifie que tout apprenant voit les autres apprenants de façon positive, est prêt à partager, échanger des idées, donner et trouver du soutien. Un apprenant autonome est responsable de sa formation, il a choisi de se former et il est motivé à réussir, il est capable de faire des choix, de demander de l'aide, de gérer son temps et a intégré des méthodes de travail. 
Ces compétences sont primordiales pour la réussite d'un apprentissage. Viviane Glikman (2001, p. 63) parle de la motivation et de l'autonomie chez l'apprenant comme étant un facteur-clé pour la réussite de son apprentissage à distance : « Au total, on constate que, même si beaucoup d'apprenants estiment très important et rassurant de savoir qu'un tutorat est disponible et qu'ils peuvent éventuellement y recourir, seuls les déterminés s'adressent spontanément aux tuteurs, alors que leurs besoins en termes de soutien ne sont pas les plus cruciaux. Ils représentent généralement, semble-t-il, une faible proportion des adultes en formation (entre 10 et $25 \%$ selon le dispositif considéré), ce qui explique la faible utilisation du tutorat lorsque celui-ci exige une initiative de la part des apprenants. »

Il s'agit bien là des compétences métacognitives mises en évidence également par Claire Bélisle et Monique Linard (1996). Celles-ci sont mises à contribution avec plus d'insistance dans le cadre de l'EAD (Enseignement à Distance). En effet, si elles font défaut, ce manque se ressent davantage. Il est alors chiffré en termes d'échecs et d'abandons.

Le soin, la précision, la complétude, tout ce qui participe de la qualité des contenus est essentiel, mais un environnement d'EAD qui ne porterait pas une attention particulière à l'aptitude à apprendre à distance ne conviendrait qu'aux «bons élèves». Nous mettons de coté l'aptitude à utiliser les TIC, considérant qu'en licence informatique cette aptitude est déjà plus ou moins développée chez des futurs informaticiens, ou qu'elle le sera rapidement.

\section{Une démarche collaborative}

Le groupe est composé des apprenants et d'un tuteur. Comme le montrent France Henri et Karin Lundgren-Cayrol (2001), l'apprentissage collaboratif nous semble adapté à un groupe d'adultes. Cette affirmation s'appuie sur des théories de l'apprentissage recoupant les valeurs du constructivisme et les principes andragogiques. L'apprentissage collaboratif offre un cadre de soutien mutuel tout en demandant une démarche active de l'apprenant responsable de son apprentissage. D'après ces auteures, «le profil recherché pour l'apprenant collaboratif recoupe celui que propose l'andragogie et qu'elle décrit comme étant celui de l'adulte qui, en raison de son expérience et de sa situation de vie, apprend de manière plus autonome que les plus jeunes...» (p. 28). Il est intéressant également de ne pas parler seulement de collaboration, mais d'une démarche collaborative. On attend en effet de l'apprenant une attitude face à l'apprentissage dans laquelle il construit ses connaissances en lien avec le groupe. Cette attitude l'amènera à travailler en groupe tout en gardant la responsabilité de ses stratégies d'apprentissage en autoformation. Cette démarche préconisée pour réussir une formation à distance n'est pas simple à réaliser avec tous les apprenants.

On peut penser qu'un certain nombre d'apprenants a choisi une formation à distance suite à une non-adaptation au système scolaire traditionnel. Cette difficulté d'intégration se répercute dans leur formation à distance. Nous observons que 
parfois en choisissant ce type de formation, certains choisissent le retrait. Tout échange est une offre dont l'apprenant a la liberté de se saisir ou non. Tout en invitant, en incitant, en motivant les apprenants à échanger, nous ne posons pas comme obligatoire les échanges dans la communauté des apprenants et avec le tuteur. Il y a aussi les apprenants qui souhaitent échanger parce qu'ils croient au soutien moral du groupe et à une construction collective des connaissances. Cependant, même parmi ceux-ci, certains éprouvent des difficultés à s'y consacrer. Reste le problème de la disponibilité des apprenants à distance, qui cumulent presque tous une activité professionnelle avec leur formation.

Considérant qu'un adulte prend la responsabilité de ses stratégies d'apprentissage, nous proposons un cadre dans lequel les échanges sont favorisés tout en restant optionnels et souples. Néanmoins, l'action du tuteur est importante pour renforcer ce cadre. Son rôle est présenté dans le paragraphe suivant.

\section{Le rôle du tuteur}

\section{Développer des capacités métacognitives}

Le rôle du tuteur va au-delà d'une capacité à traiter des contenus (valider une réponse, donner des éléments de réponse sur les savoirs). Certes, le tuteur est tenu de maîtriser les connaissances transmises, d'autant plus nécessairement qu'il a besoin de se rendre disponible pour porter son attention sur le processus d'apprentissage des apprenants. En effet, le rôle du tuteur est de fournir un soutien à l'apprenant tant du point de vue cognitif que métacognitif. Il a, par exemple, à soutenir sa motivation dans un contexte où le découragement est fréquent. Un facteur déterminant pour la réussite d'un apprentissage à distance est le développement individuel et collectif de compétences métacognitives. Nous ne développons pas davantage ici ces compétences, qui relèvent par exemple de l'écoute, la sécurité, l'appartenance au groupe, la motivation... Henri et Lundgren-Cayrol (2001, p. 114) déclinent les interventions qui stimulent le sentiment d'appartenance au groupe.

Quelle est la place du tuteur dans un contexte de travail collaboratif ? Dans un contexte d'apprentissage où l'on privilégie la collaboration, la place du tuteur n'est pas au centre. Nicolas Michinov (2005, p. 98) précise que «... le formateur passe d'un rôle 'd'acteur principal' sur la scène de la formation à un rôle de 'metteur en scène' chargé de scénariser les activités en ligne (ou de mettre en œuvre un scénario) et d'accompagner les groupes d'apprenants en les guidant tout au long de leur parcours de formation ».

Dans le chapitre sur les communautés virtuelles d'apprentissage, Pierre Dillenbourg, Charline Poirier et Laure Carles (2003, p. 29) soulignent qu'il est important de ne pas «... rendre cette communauté trop formelle. Le manque de flexibilité, les structures et règles trop formelles sont des obstacles à la survie d'une communauté. » 
Le tuteur est un acteur parmi d'autres au sein du groupe, mais il est aussi un acteur particulier en tant qu'expert sur des contenus et en tant que pédagogue. C'est une personne-clé pour animer et modérer les actions collaboratives (forum, travaux en groupe...).

Il doit faire preuve de souplesse car il est important aussi qu'il laisse vivre le groupe, qu'il accueille les initiatives et tolère l'existence d'échanges non contrôlés.

\section{Amplifier le geste pédagogique}

Une condition majeure pour que le tuteur intervienne sur des aspects relevant de la métacognition est d'inscrire cette intervention dans une forte interaction avec les apprenants.

Dans un environnement d'apprentissage à distance, au sein duquel les échanges s'effectuent essentiellement par écrit, quelle qualité de relation peut-on développer ?

Cette question pose le problème suivant: comment faire passer au mieux un message lorsque seule la communication écrite fonctionne ? Le non écrit concerne la communication non verbale : visage, attitude, ton de la voix... Ce besoin de dire au-delà des mots se ressent par l'apparition des smileys, faible compensation.

Prenons un exemple. En situation de face à face, un élève plutôt discret d'habitude, prend la parole. L'enseignant l'encourage, lui sourit, l'invite à continuer, l'aide à trouver ses mots... Au cours suivant, un regard accueillant peut suffire pour inviter l'élève à prendre à nouveau la parole. Ce geste pédagogique adapté à la situation a des chances d'être efficace.

Cet exemple montre deux choses :

- l'enseignant a une intention pédagogique lorsqu'il adopte des modes d'intervention appropriés à une situation de retrait. Nous appelons cela un geste pédagogique ;

- ce geste pédagogique s'effectue de façon verbale et non verbale (un regard accueillant).

C'est le deuxième point qui nous intéresse ici.

L'écrit peut être un atout quand il s'agit pour le tuteur de réfléchir à un diagnostic et à la façon d'y répondre, car la trace écrite des échanges facilite cette analyse. De plus, la propriété asynchrone des échanges laisse le temps de la réflexion.

Mais revenons sur la question de la communication pour savoir comment le tuteur à distance peut compenser l'absence de communication non verbale sur un dispositif où les interactions sont essentiellement réalisées par courriels. Au thêâtre, les acteurs sont à une certaine distance du public. Pour faire passer un geste, ils l'amplifient. S'ils sourient, ils exagèrent le sourire afin qu'il soit perçu. De la même façon, sur la scène de l'EAD, le tuteur doit exagérer son geste pédagogique. Par 
exemple, suite à une question posée par un apprenant, il ne doit pas seulement se contenter de répondre sur les savoirs en terminant par «à bientôt » ou « cordialement ». Si l'apprenant est habituellement plutôt silencieux, il est important que le tuteur complète sa réponse en pointant le fait qu'il est le bienvenu avec ses questions, que toute question est bonne, qu'il a la possibilité d'utiliser le forum ou d'autres ressources d'échanges. Si la question s'y prête, il peut envisager de la publier sur le forum. Cette dernière possibilité doit toutefois être exploitée avec prudence, car une publication écrite reste et est vue par tous. Autre exemple, le tuteur ne doit pas négliger des phrases comme «mais, je ne suis pas sûre d'être sur la bonne voie ». Par exemple, il cherchera dans ce cas à renforcer la confiance de l'apprenant dans ses approches. Les apprenants sont isolés, ils ont à intégrer le fait que le tuteur les accompagne et que le groupe existe. Un tuteur trop modeste, trop frileux, est comme un acteur qui n'a pas compris qu'il ne doit pas sourire de la même manière sur scène comme dans la vie. Le tuteur a en charge de repérer les petites phrases qui «parlent» des métaconnaissances, il peut aussi déceler des difficultés en lisant entre les lignes. Ensuite sa réponse est un geste pédagogique qu'il exagérera pour être perçu, cherchant à optimiser son effet.

La continuité temporelle des échanges tisse une relation. C'est pourquoi cette attitude pédagogique est à développer dans la durée. Il est important aussi que le tuteur s'adresse fréquemment au groupe, par exemple dans une rubrique « actualités », parfois juste pour maintenir le lien, en rappelant une date...

Cette exagération du geste pédagogique, pour être efficace, doit être exercée de façon subtile, le geste est un soutien actif, positif et présent dans le lien. Il est alors opportun de suivre les étudiants en difficulté en les interpellant. Agir au bon endroit et le faire en nuances est un art que le tuteur doit exercer dans la relation. C'est pourquoi il est essentiel qu'il possède des compétences relationnelles ou qu'il soit formé pour les acquérir.

\section{Les outils informatisés pour l'apprentissage à distance}

Nous présentons ici un outil informatisé développé dans le cadre de nos activités d'enseignement à distance (cf. supra «La licence informatique à distance »). Il s'agit d'un exerciseur, c'est-à-dire d'un système informatique mettant des exercices d'application à la disposition des étudiants.

Nous centrons la présentation de cet exerciseur sur les choix conceptuels qui ont présidé à sa création, car sa conception s'inscrit dans une approche pédagogique visant à améliorer l'environnement d'apprentissage à distance. Il est en effet important de montrer, à l'heure où se discute l'industrialisation du tutorat, en quoi les outils informatisés peuvent soutenir, voire faciliter, des orientations pédagogiques liées au rôle du tuteur. 


\section{Un exerciseur pour l'enseignement à distance}

Cet exerciseur complète d'autres supports pédagogiques tels que le cours en ligne comprenant des petits exercices d'intégration et un parcours pédagogique proposant des exercices de travaux dirigés et trois devoirs à rendre. Le but de cet outil est de permettre à l'apprenant d'élargir son expérience dans la programmation par la conception et l'implantation de nouveaux programmes. Il invite l'apprenant à réviser sa pratique de la programmation, à intégrer des points de méthodes et à s'auto-évaluer. Cet outil est particulièrement adapté à la formation aux métiers de la programmation car devenir programmeur requiert une pratique importante sur machine.

\section{Une base de données d'exercices}

Pour tenir compte de l'hétérogénéité des niveaux, chaque exercice affiche un degré de difficulté. Ainsi chaque apprenant peut s'exercer en fonction de l'estimation de ses capacités. L'indication des niveaux lui permet de s'auto-évaluer. Cet exerciseur comprend donc une base de données d'exercices organisés par thème et par niveau de difficulté.

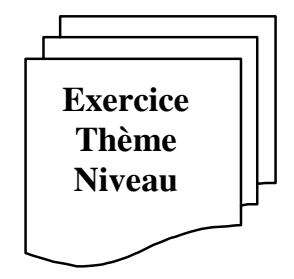

Figure 1. Exerciseur : base de données des exercices

Les TIC offrent d'énormes possibilités de stockage, de reproduction et de distribution de l'information. On aurait pu se contenter de la base de données, en ajoutant simplement une interface afin que l'apprenant accède aux exercices organisés par thème et par niveau. Cet outil, déjà bien utile, ne tiendrait alors pas compte des particularités inhérentes à l'acquisition des connaissances dans le cadre d'un enseignement à distance.

Notre expérience nous montre que la seule existence d'un outil ne suffit pas pour que l'apprenant l'utilise. Un forum de discussion est implanté sur le campus virtuel que nous utilisons depuis cinq ans, mais c'est seulement depuis que le tuteur anime ce forum qu'il est devenu support d'échanges et lieu de rendez-vous. On retrouve ce constat dans d'autres expériences d'enseignement à distance. Michinov (2005, p. 96), rapporte que : «... ce n'est pas parce qu'un dispositif technique permet la mutualisation des connaissances que ces connaissances seront mutualisées. De la même façon, ce n'est pas parce que des outils techniques permettent à des personnes 
géographiquement dispersées de collaborer, qu'elles collaboreront. On se heurte à l'illusion communément répandue selon laquelle les membres d'une communauté vont échanger, partager et collaborer parce qu'un environnement technique le permet. Notons que l'on retrouve cette illusion dans la conception des outils ellemême où le déterminisme technologique prime souvent sur le déterminisme social relatif aux usages. »

Concernant l'usage des ressources mises à disposition des apprenants, le tuteur a un rôle important à jouer pour enclencher l'utilisation d'un outil qui dépasse une première curiosité et soit réellement active. Dans le cadre de l'utilisation de l'exerciseur, le tuteur est expert en ce qui concerne les contenus et, à ce titre, il a la possibilité d'inciter l'apprenant à s'exercer en le renvoyant à un exercice bien ciblé en fonction d'une question posée. Ensuite, il continue d'accompagner l'apprenant et revient avec lui sur l'expérience vécue en termes de bilan. En effet, si les outils aident l'apprenant à progresser, le tuteur ne doit pas minimiser son rôle pour autant. Il doit jouer un véritable rôle d'animateur autour de l'utilisation des outils, sinon ces derniers ne «vivent» pas. Rappelons qu'à distance il est «facile» pour un apprenant de se décourager et de perdre pied. Il se fait alors oublier et ceux qui ont tendance à ne pas s'exprimer s'isolent très rapidement. Dans l'optique de limiter cet isolement, le fait de valoriser les expériences au sein de l'ensemble du groupe est porteur aussi. Il est important que cette activité d'animation soit également relayée par l'ensemble des membres du groupe. Le point suivant montre comment favoriser ces échanges.

\section{L'appartenance au groupe}

Dans l'ouvrage collectif Pédagogies.net (Taurisson et Sentini, 2003), Aude Dufresne cherche à définir des critères de réussite qui doivent guider la conception de systèmes d'apprentissage. Dans un paragraphe consacré à la rétroaction (p. 152), elle note que «si l'on considère les communautés d'apprentissage, on constate que la rétroaction y est souvent très pauvre. Qui est ce groupe auquel j'appartiens ? Qui est en ligne ? Sur quoi les autres travaillent-ils ?... La conscience du groupe est très difficile à jauger sans cette rétroaction ».

Afin de favoriser le rôle du groupe que nous considérons comme moteur, nous avons complété l'exerciseur par des fonctionnalités utiles pour que l'apprenant se sente appartenir à un groupe. Ces fonctionnalités servent à stimuler et valoriser la collaboration.

Ainsi, la base de données est complétée par :

- un historique des connexions qui permet à un apprenant de savoir qui a «cliqué » sur le même exercice et quand. Sachant qui est susceptible de s'être intéressé à un exercice, voire de l'avoir approfondi, il peut, si besoin, échanger sur un type d'exercice avec ses collègues apprenants ; 
- la possibilité, pour un apprenant qui rencontre des difficultés pour résoudre un exercice, de poser une question qui sera automatiquement communiquée aux autres apprenants qui ont «cliqué » sur cet exercice et qui ont accepté d'être contactés sur ce sujet.

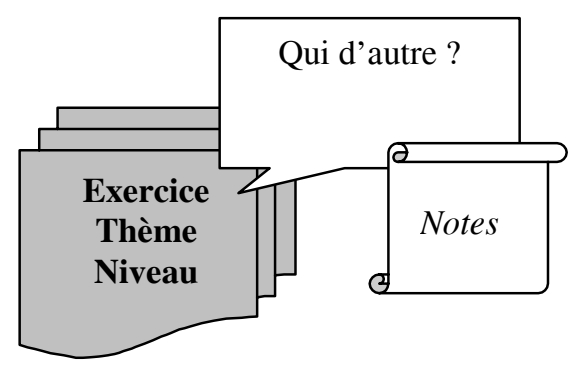

Figure 2. Exerciseur : je suis avec qui?

Cette option vient renforcer l'appartenance au groupe et répond partiellement au besoin de sécurité de l'apprenant. Nous cherchons à l'encourager à partager ses questions et ses connaissances avec les autres membres du groupe.

Les étudiants ont également la possibilité de rédiger une note à l'issue de chaque exercice. Ce commentaire les amène à préciser les apports de cet exercice et ses défauts éventuels (manque de clarté...), ils peuvent apporter des compléments à sa rédaction. Ainsi les contenus ne sont pas uniquement issus de la tête et de la pratique du formateur. Ces possibilités permettent de constituer une mémoire des discussions, des bonnes questions, des solutions apportées. L'environnement d'apprentissage se modifie, par conséquent, en fonction des interventions des apprenants. Les connaissances évoluent avec les contributions du groupe.

\section{Les métadonnées}

Afin d'offrir à l'apprenant la possibilité de se prendre en charge dans le choix des exercices à traiter et d'orienter son travail en fonction d'un objectif donné (par exemple, un devoir à rendre), les exercices sont classés par mots-clés. La façon de renseigner les ressources pédagogiques est essentielle car, à distance, il est primordial que l'apprenant ait de bons repères dans la masse d'informations livrées. Un soin particulier est donc porté au choix de mots-clés précis.

L'apprenant peut accéder aux exercices par plusieurs entrées :

- les thèmes ;

- les mots-clés ;

- en fin de chapitre, la liste des exercices s'y rapportant ;

- deux lignes de présentation. 

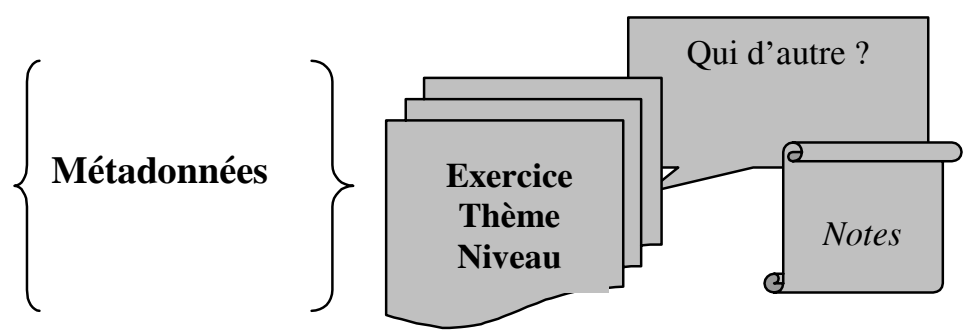

Figure 3. Exerciseur : sélection et portes d'entrée des exercices

L'indexation des données exige un important travail de réflexion sur les liens sémantiques reliant les contenus. Une bonne indexation facilite également la réutilisation des contenus et participe donc à ce que l'étudiant «rencontre » ce contenu.

Le fait de multiplier les points d'entrée (métadonnées) des objets de l'apprentissage nous semble essentiel, car l'apprentissage passe par des recoupements, par plusieurs chemins, parfois par une simple curiosité... A ce titre, il est bon de proposer plusieurs voies pour atteindre un objet d'apprentissage. Pour autant, nous ne sommes pas en train de préconiser un tutoriel trop complexe, mais plutôt un dispositif proposant une variété de ressources reliées entre elles. Il est évident qu'une charte graphique bien conçue et cohérente sur l'ensemble des objets reste un facteur de qualité, car cette cohérence participe de la sécurité de l'apprenant, d'un ancrage affectif aussi, lorsque l'apprenant retrouve un environnement familier. Nous apportons seulement de la souplesse par la multiplication des objets et des chemins pour les atteindre. Cette variété est reprise dans le point suivant (les aides) quand nous soulignons l'importance de redire autrement les mêmes notions.

\section{Les aides}

Les questions qui s'y prêtent, et c'est le cas de la plupart d'entre elles, sont assorties d'aides pour les résoudre. Ces aides renforcent, en les mettant en relief, les éléments importants comme, par exemple, des notions de cours ou des points de méthode utiles pour trouver une solution. Cette façon de focaliser l'attention de l'apprenant est importante dans la répétition, sous un autre éclairage, des contenus et des approches associées.

Il est en effet absolument primordial, pour faciliter leur intégration, de redire autrement les mêmes choses et de les dire dans plusieurs contextes de travail.

L'occasion est ici donnée de faire une parenthèse sur la répétition. Le tuteur n'est pas le seul à s'exprimer sur les solutions. Il est important qu'il laisse la parole au groupe, voire même qu'il reprenne le vocabulaire, le style des apprenants, pour 
redire les choses, par exemple dans un forum de discussions. Rappelons que le public est plus hétérogène quand l'enseignement est effectué à distance. Plutôt que de voir cette pluralité comme une difficulté pédagogique, on peut la transformer en un atout pour le groupe.

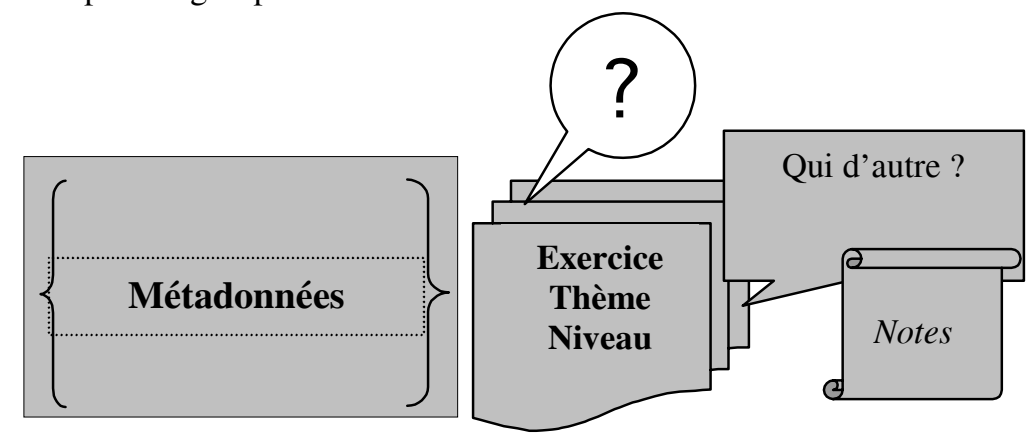

Figure 4. Exerciseur : une intégration par les aides

\section{Un parcours personnalisé tenant compte du degré de réussite}

Nous avons établi une typologie des erreurs les plus fréquentes. Celle-ci nous a guidé pour définir les aides, mais également pour confronter l'apprenant à ses erreurs. Lorsque celui-ci a terminé un exercice, une solution type lui est proposée. Par comparaison avec ce qu'il a produit, et guidé par un questionnaire, il peut en déduire les points sur lesquels il doit revenir. Nous insistons sur une prise en charge de l'apprenant par rapport à ses difficultés. Le tuteur peut simplement l'accompagner dans son diagnostic en lui proposant d'exprimer ses lacunes avec lui, par exemple pour en déduire une liste de mots-clés. Ces points à revoir renvoient alors sur des exercices ciblés et sont également reliés à des explications sur les erreurs les plus couramment rencontrées. Le fait de pouvoir lister les erreurs fréquentes est de surcroît très utile pour une auto-évaluation.

Bien évidemment, nous ne manquons pas de féliciter un apprenant qui a réussi un exercice et nous l'invitons à passer à d'autres étapes dans son apprentissage. Ainsi l'apprenant construit un parcours personnalisé à travers l'ensemble des exercices proposés en fonction de son niveau.

Une option supplémentaire permet de constituer, automatiquement et à tout moment, un résumé du parcours de chacun. L'historique de l'enchaînement des activités des apprenants intéresse l'apprenant et le tuteur en termes de bilan intermédiaire avec des remédiations possibles.

Au terme de la description de la conception pédagogique de l'exerciseur, nous présentons les conditions de son bon fonctionnement qui en constituent également ses limites. 


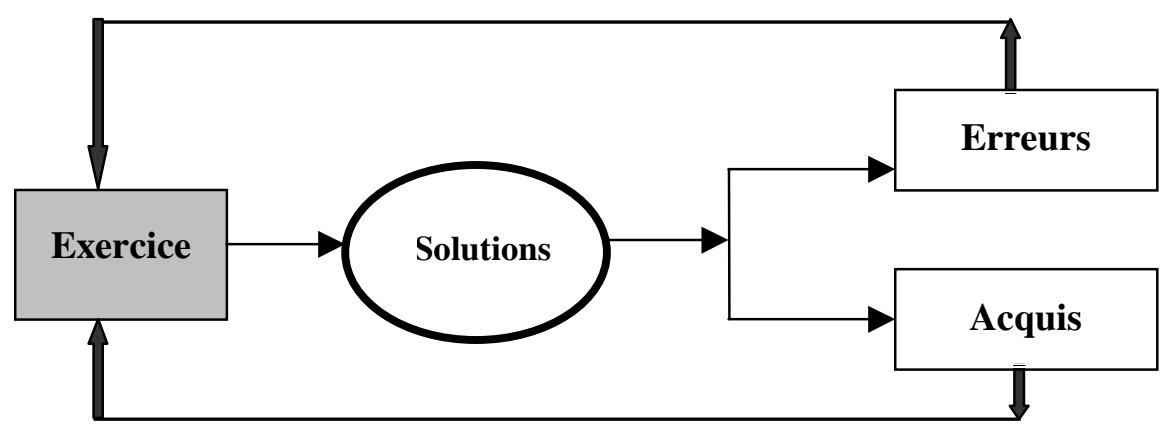

Figure 5. Exerciseur : un parcours personnalisé

\section{Limites de ce dispositif}

L'exerciseur est un exemple d'outil fait sur mesure pour l'enseignement à distance, car il est conçu en relation avec les particularités du contexte pédagogique dans lequel il est implanté. Nous avons cherché à créer un support pour apporter des solutions à des problèmes pédagogiques identifiés (auto-évaluation, appartenance au groupe, collaboration...).

Cet outil est effectivement conçu pour faire davantage que proposer des exercices, nous avons cherché à ce qu'il incite les apprenants à collaborer. Par exemple, lorsqu'un apprenant travaille sur un exercice, il dispose d'informations sur les noms, dates, heures et durées des autres connections sur ce même exercice. Il peut aussi consulter les notes prises par les autres apprenants en fin de travail. On imagine alors que l'étudiant va communiquer avec ses pairs pour poser une question en cas de difficulté, pour répondre à une question ou pour compléter les notes déjà prises. Cependant, cette forme de collaboration s'instaurera et sera maintenue au cours de l'apprentissage si, et seulement si, une grande majorité des apprenants est et se sent engagée à collaborer.

Cet engagement est bien une attitude, qui est liée à la personnalité des apprenants. Les capacités et la volonté de collaborer ne font donc pas forcément partie des prérequis dans la communauté des apprenants. Nous savons que, par ailleurs, les apprenants à distance ne disposent pas toujours de beaucoup de temps.

Nous touchons là aux limites du système proposé. Nous constatons fréquemment un essoufflement des échanges au fil des semaines. Sur un soixantaine d'étudiants, une dizaine participe régulièrement aux discussions avec quelques leaders, alors que d'autres (une dizaine également) «se perdent» en cours de route. Il reste ceux qui ne participent pas. Qu'en est-il de ceux qui ne s'expriment pas? Il y a ceux qui ont 
choisi de travailler seuls, ceux qui éprouvent de réelles difficultés de communication et aussi ceux qui ne sont pas motivés ou mal orientés et qui abdiquent vite.

Pour faciliter la collaboration, il est primordial que collaborer passe d'abord par un contrat pris dans le groupe. En début de module, nous rencontrons les apprenants environ une heure et demie, en présentiel. Cette rencontre est importante pour la suite. C'est à ce moment-là que les modalités de fonctionnement sont présentées et qu'un contrat est établi entre eux et le tuteur, et entre chacun d'eux et le groupe. Ce contrat demande un degré minimum d'implication de l'apprenant. Cet engagement fait suite à une discussion dans laquelle les modalités sont discutées pour aboutir à une prise de décision collective de l'engagement et des moyens pour le tenir.

Cette démarche de collaboration est aussi relayée par le tuteur et soutenue par le groupe d'apprenants qui participent à ces activités. Tout au long de l'apprentissage, le tuteur doit être attentif à ce que la collaboration existe et s'inscrive dans la durée. Ces gestes pédagogiques viseront à maintenir la motivation individuelle, la dynamique de groupe, la valorisation des échanges, l'incitation à utiliser les ressources proposées. En tant qu'expert et pédagogue dans le groupe, le tuteur est écouté d'une oreille attentive par les apprenants. Il peut, par exemple, valoriser toute démarche collaborative en publiant et en validant des résultats issus de celle-ci. Il est important qu'il nomme (avec leur accord) les étudiants qui y participent afin de les valoriser aux yeux du groupe. Il travaille ainsi en s'appuyant sur les personnes du groupe qui collaborent.

La première version de cet exerciseur a été mise en ligne à la rentrée 2004-2005 pour une soixantaine d'étudiants. A travers un premier bilan de cette expérience, nous constatons que les étudiants ont utilisé l'outil de façon "épisodique », même s'ils soulignent l'intérêt des aides associées aux questions, la commodité de l'indexation des exercices et leur satisfaction face aux échanges que l'outil suscite. Une amélioration certaine résiderait dans le développement d'une interface hommemachine plus agréable et plus ergonomique. Dans sa première version, l'interface de l'exerciseur reste assez élémentaire. Il est également nécessaire que la base de données renferme un bon nombre d'exercices pour que chaque apprenant y trouve un (ou des) intérêt(s). L'amélioration de ces deux points nous permettra de proposer une nouvelle version de l'exerciseur intégrant un potentiel de travail collaboratif.

\section{Industrialisation du tutorat}

La présentation d'un outil informatisé pour l'EAD et des fonctions tutorales attendues dans le cadre de notre pratique nous apportent un certain éclairage sur la question de l'industrialisation du tutorat.

Nous avons montré que, pour que ses gestes aient une portée, le tuteur doit mettre son énergie et son professionnalisme pour les amplifier. Il s'agit de les grossir, compte tenu des canaux de communication mis à disposition pour enseigner 
à distance via Internet. Nous pensons que les outils informatisés participent eux aussi à cette amplification. Par exemple, l'amplification de l'incitation à collaborer passe par une des fonctionnalités de l'exerciseur qui consiste à envoyer automatiquement une question aux étudiants qui ont déjà consulté le même exercice. Ainsi, l'outil d'apprentissage, en relayant le rôle du tuteur, vient renforcer ses intentions. A ce titre, il amplifie le geste pédagogique du tuteur. Bien évidemment, forcer la collaboration n'est pas une bonne solution. Afin que leurs rythmes de travail soient respectés, les apprenants se déclarent eux-mêmes volontaires ou non pour collaborer à l'issue d'un exercice. Le tuteur peut toutefois être amené à rappeler le contrat d'engagement et à valoriser la participation. La dynamique de groupe reste une entreprise délicate. Raison de plus pour encourager la mise en place de moyens informatisés développés pour renforcer la démarche collaborative.

Le travail de conception de l'exerciseur, étroitement lié à une réflexion sur notre pratique, nous permet d'avancer l'hypothèse qu'une utilisation réussie de ce type d'outil tient à la présence et à la qualité de facteurs tels que de bons rapports affectifs avec l'apprentissage, la définition de diagnostics précis, le sentiment d'appartenance au groupe, le balisage du parcours individuel et collectif... Nous trouvons d'autres outils pour l'apprentissage qui développent des facteurs de qualité de ce type. Par exemple, l'outil CUMULI (Construction d'Une Mémoire du groUpe dans L'Interaction en FAD) est «un incitateur à la communication asynchrone prenant en compte le niveau d'intérêt et de compétence de chaque membre du groupe pour chaque thème discuté.» (Reffay et Chanier, 2001, p. 155). Il est intéressant de noter qu'une spécificité de l'EAD comme l'isolement de l'apprenant peut, avec un tel outil, être partiellement palliée, voire devenir un atout.

En résumé, selon nous, un bon couplage des intentions pédagogiques et des fonctionnalités de l'outil demeure un garant pour atteindre les bénéfices escomptés. Proposer des outils d'apprentissage conçus pour servir et développer la contribution du tuteur et la collaboration de groupe nous semble être une voie réaliste et efficace.

Evoquant les questions de standardisation et de normalisation, J.-P. Pernin (2004, p. 44) explique que « dans un champ complexe où le facteur humain tient un rôle primordial, les premières propositions centrées sur la technique ont eu davantage pour conséquence la limitation des approches pédagogiques que leur enrichissement. Même si nous savons que le chemin sera long, il nous semble primordial de poursuivre les efforts pour disposer de modèles communs permettant l'émergence de nouvelles solutions et le renforcement de la diversité des approches pédagogiques. »

Nous partageons l'idée selon laquelle les nouvelles technologies ne doivent pas être mises en avant lors de la conception de ressources pédagogiques, mais plutôt au service de la pédagogie et de la complexité des processus d'apprentissage. C'est dans cet esprit que l'utilisation des nouvelles technologies liées au développement d'Internet est puissante et précieuse et vient réellement servir un enseignement à distance de qualité. 


\section{Conclusion}

En considérant qu'industrialiser le tutorat c'est constituer des machines pour apprendre, cette opération demande de définir un modèle pédagogique, puis d'analyser comment le rendre opérationnel techniquement.

Notre expérience de tutorat nous conduit à constater quotidiennement qu'il est difficile de mettre à jour une véritable modélisation des pratiques pédagogiques. Nous remarquons que l'essentiel de l'action menée par le tuteur, dans son rôle d'accompagnateur et de guide, consiste à agir sur les processus d'apprentissage de l'apprenant. Ce type d'intervention s'effectue dans une dynamique de relations et d'échanges. A distance, le tuteur accomplit un véritable geste pédagogique, en construisant une présence, en développant des rapports humains. La personne tuteur est, en effet, engagée dans une dynamique d'échanges tissée d'intentions pédagogiques, assorties d'une capacité à établir des diagnostics, à écouter, à donner et à recevoir, à stimuler le plaisir d'apprendre, à user de doigté pour intervenir sur des points sensibles (la relation de l'apprenant à soi, aux autres, la confiance, la dévalorisation...). Au demeurant, ce geste pédagogique repose sur des conceptions, sur des valeurs et sur le sens que donne la personne tuteur à la formation. Elle accomplit un geste professionnel et humain. C'est pourquoi nous ne pensons pas que ce geste soit industrialisable.

Notre conception de l'apprentissage est que, pour chacun, c'est un chemin tissé d'intentions, de relations, d'affects, de gestions mentales... C'est un parcours individuel qui prend un sens différent et plus ou moins créatif selon les individus. Dans ce cadre-là, un tutorat complètement industrialisé nous semble réducteur et largement critiquable, car il ne favoriserait que les apprenants déjà autonomes, sécurisés...

Toutefois, s'il est vrai que l'ensemble des besoins pédagogiques auxquels répond un tuteur ne nous semble pas industrialisable, des outils informatisés pour l'apprentissage s'avèrent très utiles, pour l'apprenant et pour le groupe, s'ils intègrent des fonctionnalités qui soutiennent le travail d'accompagnement accompli par le tuteur. De plus, tenant compte des difficultés des apprenants liées spécifiquement au fait que l'enseignement qu'ils suivent s'effectue à distance, l'existence de tels outils constitue réellement un facteur supplémentaire de réussite.

Rappelons enfin que l'expérience nous a montré qu'un des points les plus délicats à améliorer est de développer davantage les échanges au sein du groupe.

Notre approche pragmatique et le recul sur cinq promotions formées nous indiquent que les outils informatisés, pour être réellement utilisés et apporter les bénéfices escomptés, doivent intégrer des pratiques pédagogiques favorisant par exemple une auto-évaluation et agissant sur la sécurité de l'apprenant, sa motivation à apprendre et à échanger avec ses pairs. Autrement dit, il est important de créer des outils informatisés basés non uniquement sur la transmission de savoirs, mais d'élargir leur conception pédagogique en intégrant des fonctionnalités liées au 
processus d'acquisition des connaissances et tenant compte des particularités de l'enseignement à distance. Ces fonctionnalités, en favorisant par exemple le travail collaboratif entre apprenants pour un exercice, viendront soutenir et développer une démarche d'accompagnement exercée par le tuteur en lien avec le groupe. C'est dans cet esprit que nous avons conçu un exerciseur pour l'enseignement de la programmation.

Par ailleurs, reprenant l'exemple de l'exerciseur, nous savons que si le tuteur se contente de signaler qu'il y a un exerciseur pour intégrer ou réviser des notions, cet outil sera peu ou pas du tout utilisé. En effet, il faut que les étudiants se sentent motivés et encouragés dans des besoins réels, qu'ils voient les bénéfices qu'ils en tireront, qu'ils se vivent inclus dans un groupe qui profite de cet outil, qu'ils se sentent sécurisés. Le tuteur a bien à jouer un rôle d'animateur en incitant à utiliser les ressources pédagogiques de façon active et efficace, et ceci dans la durée.

De plus, une des idées majeures développée dans cet article est que, pour être efficace, le tuteur doit également amplifier ses gestes pédagogiques. La cause principale en est la «pauvreté » des canaux de communication les plus fréquemment utilisés sur Internet, ceux-ci ne transmettant que de l'écrit. Ce besoin d'amplification est une raison supplémentaire pour mettre en avant la conception d'outils informatisés venant relayer un geste pédagogique.

L'ensemble de ces réflexions nous conduit donc à préconiser des plates-formes d'enseignement à distance reposant sur un bon couplage des technologies à distance et du geste pédagogique.

Notons que la conception d'outils d'aide à l'apprentissage présente un autre intérêt non négligeable : elle conduit le tuteur, parfois un universitaire qui dans un cadre «classique » d'enseignement l'aurait fait de façon moins approfondie, à réfléchir à la pédagogie. Vrai progrès, car les plates-formes proposent des possibilités nouvelles et novatrices (par exemple, mémoire de groupe, autoévaluation...) et permettent de tenir compte de l'hétérogénéité du groupe.

Nous portons un intérêt aux travaux de concernant les EIHA (Environnements Interactifs d'Apprentissage Humain) (Crozat et Trigano, 2002 ; Pesty et al., 2001), les métaconnaissances, les rapprochements sémantiques, les systèmes conseillers (Paquette et Tchounikine, 2002) etc. car ce sont autant de points à considérer pour améliorer les performances des outils d'aide à l'apprentissage. Selon nous, plus les outils seront performants, plus le tuteur aura le temps de se consacrer à la relation pédagogique avec chaque apprenant et avec le groupe.

Il est important, pour terminer, de souligner que l'action du tuteur, telle que nous la concevons, exige de la disponibilité et requiert une formation. Il existe des formations de tuteur à distance comme le relate Anna Wetter (2003) dans un entretien avec Jacques Rodet. 
La formation au geste pédagogique pour le tuteur est cruciale, si derrière l'idée d'industrialiser le tutorat, il y a aussi celle de mettre en œuvre un tutorat de masse. Selon nous, assurer un tutorat auprès d'un nombre important d'étudiants nécessite de mettre en place des moyens humains suffisants en nombre et en qualification, afin de proposer des formations à distance avec des tuteurs disponibles et qualifiés pour que l'EAD intègre un réel apport humain, indispensable à la réussite des apprenants.

\section{Bibliographie}

Bélisle C., Linard M., «Quelles nouvelles compétences des acteurs de la formation dans le contexte des TIC ? », in «Technologies et approches nouvelles en formation », Education Permanente, $\mathrm{n}^{\circ}$ 127, 1996, p. 19-47.

Crozat S., Trigano P., «Structuration et scénarisation de documents pédagogiques numériques dans une logique de massification", Sciences et Techniques Educatives, Hermès, vol. 9, n 3-4, 2002, p. 371-408.

Dillenbourg P., Poirier C., Carles L., «Communautés virtuelles d'apprentissage : e-jargon ou nouveau paradigme?», in Taurisson A., Sentini A., dir., Pédagogies.net. L'essor des communautés virtuelles d'apprentissage. Montréal, Canada, Presses de l'Université du Québec, coll. Education Recherche, 2003, p. 11-47.

Glikman V., «Apprenants et tuteurs : une approche européenne des médiations humaines », in Choplin H., dir., «Les TIC au service des nouveaux dispositifs de formation», Education permanente, $\mathrm{n}^{\circ} 152,3^{\mathrm{e}}$ trim. 2002, p. 55-69.

Henri F., Lundgren-Cayrol K., Apprentissage collaboratif à distance. Pour comprendre et concevoir les environnements d'apprentissage virtuels, Montréal, Canada, Presses de l’Université du Québec, 2001.

Michinov N., «Une méthodologie pour la collaboration à distance », In De la mutualisation à la collaboration. Actes du colloque Réseaux Humains/Réseaux Technologiques, vol. 7, Paris, CNDP, 2005, p. 95-105. Sous presse.

Paquette G., Tchounikine P., «Contribution à l'ingénierie des systèmes conseillers : une approche méthodologique fondée sur l'analyse du modèle de la tâche ", Sciences et Techniques Educatives, Hermès, vol. 9, n 3-4, 2002, p. 409-435.

Pernin J.-P., «A propos d'objets pédagogiques», Actes du colloque "Entre technique et pédagogie : la création de contenus multimédia pour l'enseignement et la formation", Neuchâtel, Suisse, IRDP, 2004, p. 33-45.

Pesty S., Webber C., Balacheff N., «Baghera: une architecture multi-agents pour l'apprentissage humain », in Aniorte P., Gouarderes S., eds, Cognitique, Toulouse, Cepadeus Edition, 2003, p. 204-214.

Reffay C., Chanier T., «CUMULI : construction d'une mémoire du groupe dans l'interaction en FAD », in «Actes de la Conférence EIA0'2001 », Sciences et Techniques Educatives, 2001, vol. $8, \mathrm{n}^{\circ} 1-2$, p. 155-158. 
250 D\&S - 3/2005. Tutorat et logiques industrielles

Taurisson A., Sentini A., dir., Pédagogies.net. L'essor des communautés virtuelles d'apprentissage, Montréal, Canada, Presses de l'Université du Québec, coll. Education Recherche, 2003

Wetter A., Rodet J., «La formation de tuteurs à distance », Entretien, juin 2003. Disponible sur le site http://jacques.rodet.free.fr/chroniq.htm\#06 